\title{
Active vibration suppression of non-linear beams using optimal dynamic inversion
}

\author{
Sk F Ali ${ }^{1 *}$ and $\mathbf{R}$ Padhi $^{2}$ \\ ${ }^{1}$ Department of Civil Engineering, Indian Institute of Science, Bangalore, India \\ ${ }^{2}$ Department of Aerospace Engineering, Indian Institute of Science, Bangalore, India
}

The manuscript was received on 23 September 2008 and was accepted after revision for publication on 26 March 2009.

DOI: 10.1243/09596518JSCE688

\begin{abstract}
Euler-Bernoulli beams are distributed parameter systems that are governed by a non-linear partial differential equation (PDE) of motion. This paper presents a vibration control approach for such beams that directly utilizes the non-linear PDE of motion, and hence, it is free from approximation errors (such as model reduction, linearization etc.). Two state feedback controllers are presented based on a newly developed optimal dynamic inversion technique which leads to closed-form solutions for the control variable. In one formulation a continuous controller structure is assumed in the spatial domain, whereas in the other approach it is assumed that the control force is applied through a finite number of discrete actuators located at predefined discrete locations in the spatial domain. An implicit finite difference technique with unconditional stability has been used to solve the PDE with control actions. Numerical simulation studies show that the beam vibration can effectively be decreased using either of the two formulations.
\end{abstract}

Keywords: dynamic inversion, optimal dynamic inversion, non-linear structural control, non-linear beams, Euler-Bernoulli beams

\section{INTRODUCTION}

Euler-Bernoulli beam models are widely used in various real-life applications such as civil engineering structures [1], aircraft and space structures [2, 3], robotic arms [4] etc. They are often subjected to dynamic loads and often experience large deformations. Since these structures have poor damping in general, active controllers are typically needed to minimize these large deformations to a safe limit within a short time. This is one important reason why active vibration control of beams remains a topic of significant activity in current research. Beams are described mathematically as distributed parameter systems (DPS) through partial differential equations, which can be derived by classical analytical techniques [1]. In fact, control design for DPS is often more challenging as compared to lumped parameter systems in general. Such control design

* Corresponding author: Department of Civil Engineering, Indian Institute of Science, Bangalore, Karnataka 560012, India. email: sk.faruque.ali@gmail.com; skfali@civil.iisc.ernet.in problems have been studied both from mathematical as well as engineering point of views. An interesting brief historical perspective of the control of DPS can be found in $[\mathbf{5}, \mathbf{6}]$. In a broad sense, existing control design techniques for DPS can be attributed to either 'approximate-then-design (ATD)' or 'design-then-approximate (DTA)' categories. An interested reader can refer to [7] for discussions on the relative merits and limitations of the two approaches.

In the ATD approach the idea is to first come up with a low-dimensional reduced (truncated) model, which retains the dominant modes of the system and then using this truncated model (which is often a finite-dimensional lumped parameter approximate model) to design the controller. In such an approach the partial differential equation (PDE) describing the system dynamics is mapped to a finite space resulting in a finite number of ordinary differential equations (ODEs), which is done using various methods of discretization including the lumped mass technique [2], the finite element methods [3, $\mathbf{8}]$, the finite difference technique $[\mathbf{9}, \mathbf{1 0}]$, or using 
Galerkin projection-based problem-oriented orthogonal basis functions (known as proper orthogonal decomposition) $[\mathbf{1 1}, \mathbf{1 2}$. This set of ODEs is then used to design the control action.

The ATD approach of control of beam vibration has been extensively studied in the literature. Vibration control on a lumped mass beam model has been studied by Kar et al. [2]. who reported a $H_{\infty}$-based control mechanism for a bridge tower and crane system using the first five modes of the vibration but they neglected higher frequency modes. A weighting filter which represents the upper bound of system uncertainties was used to avoid spillover instability due to the neglected modes. Yang [13], and Yang and Mote [14] have studied a frequency domain approach for discrete control formulation of an Euler-Bernoulli beam. The control of fully coupled non-linear integro-differential equation of a non-linear rotating Euler-Bernoulli beam was reported by Yang et al. [3] The finite element method was used to obtain the finite-dimensional model for designing the control system [3]. Non-linear feedback control of a EulerBernoulli beam discretized over space using a Bspline was reported by Tadi [15].

However, reducing an infinite-dimensional distributed system to a finite-dimensional system has a well-known disadvantage in the control design, that is control spillover, where the controller excites unmodelled higher-order modes, causing unwanted structural vibrations. Furthermore, a stability result generated for a discretized ODE model under a proposed control cannot be generalized to the PDE model under the same control [16].

It has been shown using a Greens function-based approach to control an Euler-Bernoulli beam in the frequency domain, that the modelling of beams as a lumped mass and controlling force based on the model may lead to instability [17]; i.e. the neglected higher-order modes could possibly destabilize the mechanical system (which is known as spillover instability). Since the actual number of modes in an elastic system is infinite, selection of the number of modes (during the construction of the discretized ODE model) is not trivial in general. On the other hand, if a large number of modes are utilized to approximate a PDE-driven system (for better accuracy), the order of the corresponding discretized ODE model is of correspondingly higher dimension. Hence, the control design algorithm becomes numerically intensive and it is not possible to implement such an algorithm in real-time applications.

In the DTA approach, on the other hand, the usual procedure is to use infinite-dimensional operator theory to come up with the control design in the infinite-dimensional space [18]. For implementation purpose, this controller is then approximated to a finite-dimensional space by truncating an infinite series or reducing the size of the feedback gain matrix, etc. An important advantage of this approach is that it takes into account the full system dynamics in designing the controller, and hence, usually performs better [7]. However, to the best of the knowledge of the authors, these operator theorybased DTA approaches are mainly limited to linear distributed parameter systems [18]. The method is computationally intensive and applied to some limited class of problems such as spatially invariant systems [19].

Recently, the analysis and synthesis conditions of the distributed control of distributed linear-parameter-varying systems have been proposed [10] for systems discretized (central difference scheme) both in temporal and spatial independent variables. The distributed parameter system was expressed by a group of subsystems each having a state space realization. Thereafter, based on the group of subsystems, a sufficient synthesis condition for the design of a distributed output-feedback controller was presented using the $L_{2}$-norm as the performance criterion. The example considered is a linear EulerBernoulli beam.

A proportional and derivative (PD) feedback control augmented with a distributed piezoelectric layer actuator control was proposed for rotating an Euler-Bernoulli beam in [20]. A linear velocity controller to allow feasible implementation and to avoid modal truncation was reported. Global stability of the system was investigated using a Lyapunov approach. Since, in this approach, the beam is discretized as a collection of links, virtually joined, the method is best suitable for linear DPS. Among other approaches the variable structure control (VSC) of a flexible robot arm has been tried using a system PDE with the controller parameter being obtained using a modal expansion of the system [4]. The problem with VSC is that the controller needs to estimate states and its first and second derivatives as well as shear force of the system, which is unrealistic for large systems. The computational aspects of displacement-based boundary control of slender beams using the Komorniks control algorithm was studied in [21]. The paper uses a very weak in space Faedo-Galerkin approximation and superposes suitable modes, therefore, the method limits itself to linear analysis of beams. 
In this paper an optimal dynamic inversion technique is proposed to control the vibrations in non-linear Euler-Bernoulli beams, the dynamics of which are governed by a PDE (second-order in time and fourth-order in space). Optimal dynamic inversion follow the DTA philosophy [22], yet it is fairly straightforward, quite intuitive, and reasonably simple. The technique relies on the principle of dynamic inversion coupled with optimization theory. The fundamental idea is to design a control action which enforces an asymptotically (rather exponentially) stable dynamics for the square integral of the error (integrated over the entire spatial domain). In addition, the formulation also guarantees a minimum norm control solution, which also does a simultaneous job of control allocation.

The technique followed in this paper computes the control action by directly utilizing the nonlinear PDE governing the system dynamics, and hence, it also avoids the spillover problem. Therefore, no approximation of the system dynamics (such as modal truncation, linearization, etc.) is needed in this method. The only approximation needed here is rather the spatial grid size selection for the control computation/implementation, which can be quite small, since the computational requirement in the proposed method is also minimal (because it leads to closed-form solutions for the control variable) [22]. The method is quite versatile i.e. in the sense that it can be applied to both linear and non-linear PDEs with either continuous or discrete control action in the spatial domain.

This paper reports the use of both a continuous control action as well as a set of discrete control actions in the spatial domain to suppress the vibration of the beam. The continuous actuator formulation has better theoretical properties in the sense that its convergence can be assured without any singularity. On the other hand, the discrete formulation has better practical significance as it can be implemented with relative ease. For both the discrete in space and continuous controllers, the proposed method has been seen to minimize the beam vibration within a short time. An exponential convergence is observed. Random initial conditions have been taken and the results are reported to show the efficiency of the proposed method. Results obtained in all cases are satisfactory and the beam vibration settles quickly without demanding high control action.

This paper is organized as follows. In section 2 , the formulation and derivation of a simply supported non-linear Euler-Bernoulli beam vibration are de- tailed. The mathematical model developed in this section is used for controller design. In section 3 continuous and discrete controllers for the nonlinear beam model are designed. Numerical results for uncontrolled and controlled dynamics of the non-linear Euler-Bernoulli beam are reported in section 4 .

\section{NON-LINEAR BEAM MODEL}

The classical problem of vibration of a simply supported elastic Euler-Bernoulli non-linear beam is revisited and a suitable non-linear control action is designed for its vibration suppression. In this section for completeness, the Euler-Bernoulli beam formulation is presented and the equations of motion are obtained using Hamilton's principle [1]. Usual approximations are introduced with regard to the truncation of non-linearities above a cubic order. The beam cross-section rotational inertia and inertial non-linearities are neglected. Furthermore, the longitudinal inertial forces are disregarded as it is very small in comparison to the transverse inertia of the beam. As a consequence of this, the motion transverse to the beam axis is decoupled from the longitudinal motion. With these assumptions, the dynamics of the system can be derived as follows.

The strain displacement and curvature displacement relation of any point on a beam undergoing a large displacement and a small rotation are given as $([\mathbf{1}-\mathbf{8}])$

$$
\varepsilon_{y}=v^{\prime}+\frac{1}{2} x^{\prime 2}+z x^{\prime \prime}
$$

where, (') represents derivative with respect to spatial variable $y$ (see Fig. 1) and $y$ and $z$ are locations along the length and depth of the beam respectively. As shown in Fig. 1, $v$ and $x$ are the displacements along the longitudinal ( $y$-axis) and transverse directions ( $z$-axis) respectively. Hamilton's principle [1] is used to obtain the equation of motion of the beam which is given by

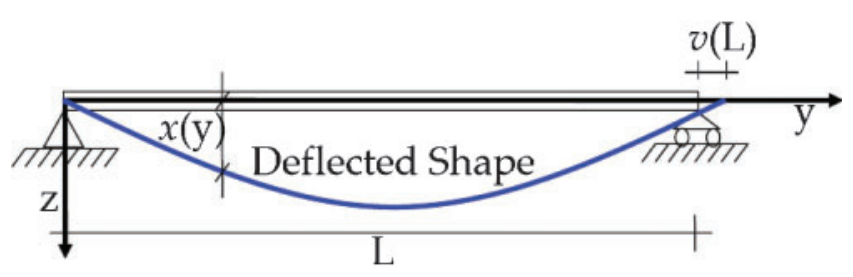

Fig. 1 Non-linear Euler-Bernoulli beam model 


$$
\int_{t_{0}}^{t}\left(\delta T-\delta \pi+\delta \omega_{\mathrm{nc}}\right) \mathrm{d} t=0
$$

where, $T, \pi$, and $\omega_{\mathrm{nc}}$ represent total kinetic, potential energy, and energy due to non-conservative forces (damping) respectively and $\delta$ denotes first variation. Neglecting the rotational inertia term, the total kinetic energy and the total potential energy are obtained as

$$
\begin{aligned}
& T=\frac{1}{2} \int_{0}^{L}\left[m\left(\dot{v}^{2}+\dot{x}^{2}\right)\right] \mathrm{d} y \\
& \pi=\frac{1}{2} \int_{0}^{L}\left[E A\left(\varepsilon_{y}^{2}+E I\left(x^{\prime \prime}\right)^{2}\right)\right] \mathrm{d} y
\end{aligned}
$$

Assuming that the damping and external force are acting along the transverse direction only. We have

$$
\delta \omega_{\mathrm{nc}}=\int_{0}^{L}(W(y, t)-2 c \dot{x}) \delta x \mathrm{~d} y
$$

where, $m, c, E, I$, and $A$ are the mass per unit length, damping, the elastic modulus, the moment of inertia, and the area of the cross-section of the beam. $W(y, t)$ represents the external force applied to the beam. First, variations of equations (3) to (5) are substituted into equation (2) and simplified. Next, the variations along the longitudinal and transverse directions are put to zero respectively to obtain the following equations of motion [8]

$$
\begin{aligned}
& m \ddot{v}-\left(E A \varepsilon_{y}\right)^{\prime}=0 \\
& m \ddot{x}+c \dot{x}+\left(E A \varepsilon_{y} z+E I x^{\prime \prime}\right)^{\prime \prime}-\left(E A \varepsilon_{y} x^{\prime}\right)^{\prime}=W(y, t)
\end{aligned}
$$

Since the longitudinal inertia is negligible in comparison to the transverse inertia $\left(E A \varepsilon_{y}\right)^{\prime}=0$ in equation (6), which results in a constant strain. It is assumed that the strain along the central axis $(z=0)$ is $\varepsilon_{0}$. Therefore, equation (6) simplifies to $v^{\prime}+1 / 2 x^{\prime 2}$. Integrating the strain along the length of the beam results in

$$
\int_{0}^{L} \varepsilon_{0} \mathrm{~d} y=\int_{0}^{L}\left(v^{\prime}+\frac{1}{2} x^{\prime 2}\right) \mathrm{d} y
$$

which on simplification gives [8]

$$
v^{\prime}=\frac{1}{2 L} \int_{0}^{L}\left(x^{\prime}\right)^{2} \mathrm{~d} y-\frac{1}{2} x^{\prime 2}
$$

Substituting equations (9) and (1) into equation (7), and then putting $z=0$, the equation for the transverse vibration of central axis of the beam becomes

$$
m \ddot{x}+c \dot{x}+E I x^{\prime \prime \prime \prime}-\frac{E A}{2 L}\left[x^{\prime \prime} \int_{0}^{L}\left(x^{\prime}\right)^{2} \mathrm{~d} y\right]=W(y, t)
$$

Equation (10) represents non-linear equation of motion of the central axis $(z=0)$ of beams with finite deformation and small rotation. The presence of the axial stiffness term in the transverse motion equation makes the system behaviour non-linear. If the axial stiffness term $\left\{(E A / 2 L)\left[x^{\prime \prime} \int_{0}^{L}\left(x^{\prime}\right)^{2} \mathrm{~d} y\right]\right\}$ is neglected then the equation of motion for a linear beam is obtained.

When $W(y, t)$ is substituted by the control force $u(y, t)$ equation (10) becomes

$$
m \ddot{x}+c \dot{x}+E I x^{\prime \prime \prime \prime}-\frac{E A}{2 L}\left[x^{\prime \prime} \int_{0}^{L}\left(x^{\prime}\right)^{2} \mathrm{~d} y\right]=u(y, t)
$$

where, $u(y, t)$ is a continuous function of both time $t>0$ and spatial variable $y \in[0, L]$ in the continuous controller case, whereas it is only a continuous function of time $t>0$ in the discrete case.

The essential boundary conditions are given in equation (12) which are true for a simply supported beam

$$
\begin{aligned}
& x(0, t)=0, \quad x^{\prime \prime}(0, t)=0, \quad x(L, t)=0, \\
& x^{\prime \prime}(L, t)=0
\end{aligned}
$$

Note that appropriate initial conditions are taken while solving and simulating the problem, which are discussed in section 4 .

\subsection{System dynamics with a continuous controller}

The system dynamics are assumed to be in control affine form and therefore the system dynamics can be written as

$$
\ddot{x}+\frac{c}{m} \dot{x}+\frac{E I}{m} x^{\prime \prime \prime \prime}-\frac{E A}{2 m L}\left[x^{\prime \prime} \int_{0}^{L}\left(x^{\prime}\right)^{2} \mathrm{~d} y\right]=\frac{1}{m} u(y, t)
$$

where the state variable $x(y, t)$ and the control variable $u(y, t)$ are continuous functions of time $t>0$ and spatial variable $y \in[0, L]$. Note that, both $x(y, t)$ 
and $u(y, t)$ are scalar functions. The boundary conditions considered are given in equation (12).

\subsection{System dynamics with discrete controllers}

The dynamical equation of motion of a non-linear beam with a set of discrete control actions, in the spatial domain is given by

$$
\begin{aligned}
\ddot{x} & +\frac{c}{m} \dot{x}+\frac{E I}{m} x^{\prime \prime \prime \prime}-\frac{E A}{2 m L}\left[x^{\prime \prime} \int_{0}^{L}\left(x^{\prime}\right)^{2} \mathrm{~d} y\right] \\
& =\frac{1}{m} \sum_{n=1}^{N} \bar{u}_{n} F\left(y, y_{n}, w_{n}\right)
\end{aligned}
$$

where the function $F\left(y, y_{n}, w_{n}\right)$ is defined as

$$
F\left(y, y_{n}, w_{n}\right) \triangleq \begin{cases}1 & \forall\left(y_{n}-\frac{w_{n}}{2}\right) \leqslant y \leqslant\left(y_{n}+\frac{w_{n}}{2}\right) \\ 0 & \text { otherwise }\end{cases}
$$

Note that the control variable appears linearly in this case too, and hence, the system dynamics are in the control affine form. It is assumed that the discrete controllers $\bar{u}_{n}$ of width $w_{n}$, are located at $y_{n}(n=1$, $\ldots, N$ ). The control action $\bar{u}_{n}$ can be shown as in Fig. 2.

The following assumptions are made for the control action.

1. In the interval $\left[y_{n}-\left(w_{n} / 2\right), y_{n}+\left(w_{n} / 2\right)\right]$, the controller $\bar{u}_{n}$ is assumed to have a constant magnitude. Outside this interval, $\bar{u}_{n}=0$. Note that the interval $w_{n}$ may or may not be small. This property is obtained by using the function $F\left(y, y_{n}, w_{n}\right)$ as defined in equation (15).

2. There is no overlapping of the controllers.

3. No controller is placed exactly at the boundary. This eliminates situations where the control action enters the system dynamics through boundary actions.

\section{CONTROL DESIGN BASED ON OPTIMAL DYNAMIC INVERSION}

The objective of the control design is to ensure that the beam vibration goes down to zero everywhere

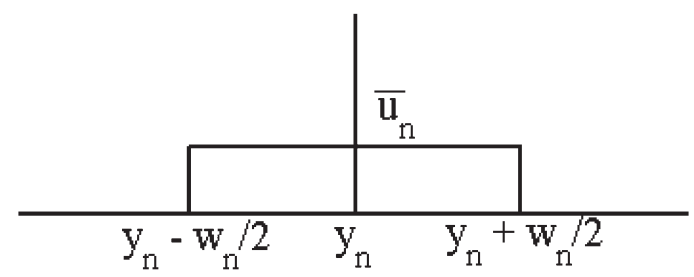

Fig. 2 Control action at $y_{n}\left(\bar{u}_{n}\right)$ along $y$ and it comes to a rest, i.e. $x(y, t) \rightarrow 0$ and $\dot{x}(y, t) \rightarrow 0$ as $t \rightarrow \infty, \forall y \in[0, L]$. To achieve our objective, an output variable (an integral error) $Z(t)$ is defined as follows

$$
Z(t)=\frac{1}{2} \int_{0}^{L}\left(\left[\begin{array}{ll}
x & \dot{x}
\end{array}\right] \mathbf{Q}\left[\begin{array}{l}
x \\
\dot{x}
\end{array}\right]\right) \mathrm{d} y
$$

where $x, \dot{x}$ are the displacement and velocity of the beam respectively, and

$$
\mathbf{Q}=\left[\begin{array}{ll}
q_{11} & q_{12} \\
q_{12} & q_{22}
\end{array}\right]>0 \quad \text { (a positive definite matrix) }
$$

is the weighing matrix, which needs to be selected by a control designer. Note that when $Z(t) \rightarrow 0$, both $x(y, t) \rightarrow 0$ and $\dot{x}(y, t) \rightarrow 0$ everywhere in $y \in[0, L]$. Using the principle of dynamic inversion $[\mathbf{2 3}, \mathbf{2 4}$, the aim of this work is to design a controller such that the following stable error dynamic is satisfied (which guarantees an exponential convergence of the evolved states to desired states)

$$
\dot{Z}+k Z=0
$$

where, $k>0$ serves as a gain. For a better physical interpretation, one may choose it as $k=1 / \tau$, where $\tau>0$ serves as a 'time constant' for the error $Z(t)$ to decay.

\subsection{Continuous control design}

In the continuous control design, equation (13) is substituted into equation (16) and then the result is substituted into equation (17). After simplification it is possible to write

$$
\int_{0}^{L}\left[\frac{1}{m}\left(q_{12} x+q_{22} \dot{x}\right) u\right] \mathrm{d} y=\gamma
$$

where

$$
\begin{aligned}
& \gamma=-\int_{0}^{L}\left[\left(q_{11} x \dot{x}+q_{12} \dot{x}^{2}\right)-\left(q_{21} x+q_{22} \dot{x}\right)\right. \\
& \times\left\{\frac{c}{m} \dot{x}+\frac{E I}{m} x^{\prime \prime \prime \prime}-\frac{E A}{2 m L}\left[x^{\prime \prime} \int_{0}^{L}\left(x^{\prime}\right)^{2} \mathrm{~d} y\right]\right\} \\
& \left.+\frac{k}{2}\left(\left[\begin{array}{ll}
x & \dot{x}
\end{array}\right] \mathbf{Q}\left[\begin{array}{l}
x \\
\dot{x}
\end{array}\right]\right)\right] \mathrm{d} y
\end{aligned}
$$

Since equation (18) is an integral equation with variable $u(y, t)$, we can have infinitely many solutions for $u(y, t) \forall t>0$ and $y \in[0, L]$. To obtain a unique and optimal solution for the control variable, a constrained optimization problem is formulated in 
which the objective is to minimize

$$
J=\frac{1}{2} \int_{0}^{L}\left[r(y)(u(y, t))^{2}\right] \mathrm{d} y
$$

subject to the constraint in equation (18). An implication of choosing this cost function is that it is desired to track the target state with minimum control effort. Furthermore, to put a relative importance on the controller action at different locations, $r(y)>0 \forall y \in[0, L]$ is introduced as a weighing function in equation (20). The particular function $r(y)$ is to be chosen by the control designer.

To use the techniques of constrained optimization [25], the following augmented cost function is formulated

$$
\bar{J}=\frac{1}{2} \int_{0}^{L} r u^{2} \mathrm{~d} y+\lambda\left\{\int_{0}^{L}\left[\frac{1}{m}\left(q_{12} x+q_{22} \dot{x}\right) u\right] \mathrm{d} y-\gamma\right\}(21)
$$

where $\lambda$ is the Lagrange multiplier, which is a free variable needed to convert the constrained optimization problem to a free optimization problem. The necessary condition of optimality [25] is given by

$$
\delta \bar{J}=0
$$

where, $\delta \bar{J}$ is the first variation of $\bar{J}$. Which gives

$$
\begin{aligned}
& \int_{0}^{L}\left[r u+\frac{\lambda}{m}\left(q_{12} x+q_{22} \dot{x}\right)\right] \delta u \mathrm{~d} y \\
& \quad+\delta \lambda\left[\int_{0}^{L} \frac{1}{m}\left(q_{12} x+q_{22} \dot{x}\right) u \mathrm{~d} y-\gamma\right]=0
\end{aligned}
$$

Equation (23) should be satisfied for all variation of $\delta \lambda$ and $\delta u$, which leads to

$$
\begin{aligned}
& r u+\frac{\lambda}{m}\left(q_{12} x+q_{22} \dot{x}\right)=0 \\
& \int_{0}^{L} \frac{1}{m}\left(q_{12} x+q_{22} \dot{x}\right) u \mathrm{~d} y-\gamma=0
\end{aligned}
$$

Solving equations (24) and (25) for $u(y, t)$ and $\lambda$ leads to

$$
\begin{aligned}
& u=-\frac{\lambda}{r m}\left(q_{12} x+q_{22} \dot{x}\right) \\
& \lambda=-\gamma / \int_{0}^{L} \frac{\left(q_{12} x+q_{22} \dot{x}\right)^{2}}{m^{2} r} \mathrm{~d} y
\end{aligned}
$$

For equal relative importance to the controller at every point in $y \in[0, L]$, it can be written that $r(y)=$ constant. In this case the controller force $u(y, t)$ becomes

$$
u=\frac{m \gamma\left(q_{12} x+q_{22} \dot{x}\right)}{\int_{0}^{L}\left(q_{12} x+q_{22} \dot{x}\right)^{2} \mathrm{~d} y}
$$

It may be noticed that when system states reach their respective goals, i.e. $x \rightarrow 0$ and $\dot{x} \rightarrow 0$, there is some computational difficulty in the sense that a zero seems to appear in the denominator of equation (28), which leads to a singularity in the control solution. However, this does not happen (see proof in the appendix).

Therefore, to avoid such numerical difficulty the control action is redefined as (see appendix for details)

$$
\begin{aligned}
& u(y, t) \\
& \quad=\left\{\begin{array}{cc}
0 & \text { if } x=0, \dot{x}=0 \\
m \gamma\left(q_{12} x+q_{22} \dot{x}\right) / \int_{0}^{L}\left(q_{12} x+q_{22} \dot{x}\right)^{2} \mathrm{~d} y & \text { otherwise }
\end{array}\right.
\end{aligned}
$$

The following points are of particular relevance.

1. It can be noted that in the development of equation (55) that there is no need for the approximation of the system dynamics to come up with a closed-form control solution. However, to compute/implement the control, there is a requirement to choose a suitable grid in the spatial domain. Hence, the proposed technique can be classified into the DTA category.

2. A finer grid can be selected to compute $u(y, t)$ since the only computation that depends on the grid size in equation (55) is a couple of numerical integrations, which do not demand intensive computations.

3. Closed-form solution of the proposed controller makes it convenient for real-time applications. The final control solution can be easily encoded on a chip as the computational requirement only involves integration over the spatial domain.

\subsection{Discrete control design}

Substituting equations (14), (15), and (16) into equation (17) and then simplifying leads to 


$$
\begin{aligned}
& {\left[\int_{y_{1}-\left(w_{1} / 2\right)}^{y_{1}+\left(w_{1} / 2\right)}\left\{q_{12} x+q_{22} \dot{x}\right\} \frac{1}{m} \mathrm{~d} y\right] \bar{u}_{1}+\cdots} \\
& \quad+\left[\int_{y_{N}-\left(w_{N} / 2\right)}^{y_{N}+\left(w_{N} / 2\right)}\left\{q_{12} x+q_{22} \dot{x}\right\} \frac{1}{m} \mathrm{~d} y\right] \bar{u}_{N}=\gamma
\end{aligned}
$$

where

$$
\begin{aligned}
& \gamma=-\int_{0}^{L}\left[\left(q_{11} x \dot{x}+q_{12} \dot{x}^{2}\right)-\left(q_{21} x+q_{22} \dot{x}\right)\right. \\
& \times\left(\frac{c}{m} \dot{x}+\frac{E I}{m} x^{\prime \prime \prime \prime}-\frac{E A}{2 m L}\left[x^{\prime \prime} \int_{0}^{L}\left(x^{\prime}\right)^{2} \mathrm{~d} y\right]\right) \\
& \left.+\frac{k}{2}\left(\left[\begin{array}{ll}
x & \dot{x}
\end{array}\right] \mathbf{Q}\left[\begin{array}{l}
x \\
\dot{x}
\end{array}\right]\right)\right] \mathrm{d} y
\end{aligned}
$$

For convenience, the following definition is made

$$
I_{n}=\int_{y_{n}-\left(w_{n} / 2\right)}^{y_{n}+\left(w_{n} / 2\right)}\left\{q_{12} x+q_{22} \dot{x}\right\} \frac{1}{m} \mathrm{~d} y
$$

Then, from equations (30) and (32) it is possible to write

$$
I_{1} \bar{u}_{1}+\cdots+I_{N} \bar{u}_{N}=\gamma
$$

However, note that equation (33) is a single equation with $N$ variables $\bar{u}_{n}, n=1, \ldots, N$ and hence there are an infinite number of solutions. To obtain a unique solution it is necessary to minimize the cost function

$$
J=\frac{1}{2}\left(r_{1} w_{1} \bar{u}_{1}^{2}+\cdots+r_{N} w_{N} \bar{u}_{N}^{2}\right)
$$

subject to the constraint in equation (33). An implication of choosing this cost function is that it is desired to obtain the solution that will lead to a minimum control effort. Note that, choosing appropriate values for $r_{1}, \ldots, r_{N}>0$ in equation (34) gives a control designer the flexibility of putting a relative importance on the control magnitude at different spatial locations $y_{n}, n=1, \ldots, N$.

To use the techniques of constrained optimization [25], it is necessary to first formulate an augmented cost function in the form of

$$
\bar{J}=\frac{1}{2}\left(r_{1} w_{1} \bar{u}_{1}^{2}+\cdots+r_{N} w_{N} \bar{u}_{N}^{2}\right)+\lambda\left[I_{1} \bar{u}_{1}+\cdots+I_{N} \bar{u}_{N}\right]
$$

where $\lambda$ is the Lagrange multiplier, which is a free variable needed to convert the constrained optimi- zation problem into a free optimization problem. The necessary condition of optimality is given by [25]

$$
\frac{\partial \bar{J}}{\partial \bar{u}_{n}}, \quad n=1, \cdots, N
$$

$$
\frac{\partial \bar{J}}{\partial \lambda}=0
$$

Expanding equations (36) and (37) leads to

$$
\begin{aligned}
& r_{n} w_{n} \bar{u}_{n}+I_{n} \lambda=0, \quad n=1, \ldots, N \\
& I_{1} \bar{u}_{1}+\cdots+I_{N} \bar{u}_{N}=\gamma
\end{aligned}
$$

Eliminating $\bar{u}_{1}, \ldots, \bar{u}_{N}$ from equation (39) using equation (38), it is possible to obtain

$$
\lambda=\frac{-\gamma}{\sum_{n=1}^{N}\left(I_{n}^{2} / r_{n} w_{n}\right)}
$$

Equations (38) and (40) lead to the following expression

$$
\bar{u}_{n}=\frac{I_{n} \gamma}{r_{n} w_{n} \sum_{n=1}^{N}\left(I_{n}^{2} / r_{n} w_{n}\right)}
$$

As a special case, if we consider $r_{1}=\ldots=r_{N}$ (i.e. equal importance is given to all controllers) and $w_{1}=\ldots=w_{N}$ (i.e. widths of all controllers are the same), we have

$$
\bar{u}_{n}=\frac{I_{n} \gamma}{\|I\|_{2}^{2}}
$$

\subsubsection{Control singularity and revised goal}

From equation (42), it is clear that when $\|I\|_{2}^{2} \rightarrow 0$ (which happens when all of $I_{1}, \ldots, I_{N} \rightarrow 0$ ) and $\gamma \nrightarrow 0$, the problem of a control singularity exists in the sense that $\bar{u}_{n} \rightarrow \infty$ (since the denominator of equation (42) goes to zero faster than the corresponding numerator). Note that if the number of controllers $N$ is large, the probability of the occurrence of such a singularity is small, since all of $I_{1}, \ldots, I_{N} \rightarrow 0$ simultaneously is a strong condition. Nonetheless, such a case may arise in a transition. More important, this issue of a control singularity will always arise when $x(y, t) \rightarrow 0$ and $\dot{x}(y, t) \rightarrow 0$ for $y \in[0, L]$ (which are the primary goals of the control design). 
This happens probably because only limited control authority is possible (controllers are available only in a subset of the spatial domain), whereas the aim of this paper is to achieve a much bigger goal of tracking the state profile $y \in[0, L]$ (something that is beyond the capability of the controllers). Hence, whenever such a case arises (i.e. when all of $I_{1}, \ldots$, $I_{N} \rightarrow 0$ or, equivalently, $\|I\|_{2}^{2} \rightarrow 0$ ), to avoid the issue of control singularity, it is proposed to redefine the goal as follows (this will be called point control).

First, define a new error vector $\boldsymbol{E}=\left[e_{1}, e_{2}, \ldots, e_{n}\right]^{\mathrm{T}}$, where $e_{n} \triangleq\left[x_{n}\right]$ (here target $x_{n}^{*}=0$ ) with $x_{n} \triangleq$ Next, design a controller such that $\boldsymbol{E} \rightarrow 0$ as $t \rightarrow \infty$. In other words, the aim is to guarantee that the values of the state variable at the node points $\left(y_{n}, n=1, \ldots\right.$, $N)$ track the zero state condition. To accomplish this goal, positive definite gain matrices $\mathbf{K}$ and $\mathbf{C}$ are selected and the following dynamics are enforced

$$
\ddot{\boldsymbol{E}}+\mathbf{C} \dot{\boldsymbol{E}}+\mathbf{K} \boldsymbol{E}=0
$$

One way of selecting the gain matrices $\mathbf{K}$ and $\mathbf{C}$ is to choose them to be diagonal matrices whose $n$th diagonal element is $k_{n}=\omega_{n}^{2}$ and $c_{n}=2 \zeta_{n} \omega_{n}$ where $\omega_{n}>0$ is the desired frequency and $\zeta_{n}>0$ is the damping ratio of the error dynamics. In such a case, the $n$th channel of equation (43) can be written as

$$
\ddot{e}_{n}+c_{n} \dot{e}_{n}+k_{n} e_{n}=0
$$

Expanding the expressions for $e_{n}, \dot{e}_{n}$, and $\ddot{e}_{n}$, then solving for $\bar{u}_{n}(n=1, \ldots, N)$ the following expression is obtained

$$
\begin{aligned}
\bar{u}_{n}=-m\{ & -\frac{c}{m} \dot{x}_{n}-\frac{E I}{m} x_{n}^{\prime \prime \prime \prime}-\frac{E A}{2 m l}\left[x_{n}^{\prime \prime} \int_{0}^{L}\left\{x^{\prime}\right\}^{2} \mathrm{~d} y\right] \\
& \left.+c_{n} \dot{x}_{n}+k_{n} x_{n}+\ddot{x}_{n}^{*}\right\}
\end{aligned}
$$

where $\ddot{x}_{n}^{*}$ is the target acceleration of the beam, which is assumed to be zero.

Combining equations (42) and (45) results in a control solution of

$$
\bar{u}_{n}= \begin{cases}m\left(\frac{c}{m} \dot{x}_{n}+\frac{E I}{m} x_{n}^{\prime \prime \prime}-\frac{E A}{2 m l}\left\{x_{n}^{\prime \prime} \int_{0}^{L}\left(x^{\prime}\right)^{2} \mathrm{~d} y\right\}-c_{n} \dot{x}_{n}-k_{n} x_{n}\right) & \text { if }\|I\|_{2}<\text { tol } \\ I_{n} \gamma /\|I\|_{2}^{2} & \text { otherwise }\end{cases}
$$

where tol represents a tolerance value. An appropriate value for it can be fixed by the control designer (a convenient way to fix a good value for it is from trial-and-error simulation studies).

\section{NUMERICAL RESULTS}

Both continuous and discrete controllers were designed to control the vibration of the simply supported flexible non-linear Euler-Bernoulli beam discussed in Section 3. The non-linearity arises from the coupling of the axial stiffness terms in the transverse motion of the beam. The equation of motion in the longitudinal direction was neglected due to the smaller inertial motion in that direction with respect to the transverse one. For numerical simulations a simply supported beam of $5 \mathrm{~m}$ length was chosen and other parameters were as given in Table 1. The uncontrolled system PDE as well as the controlled system PDE were solved using an implicit finite difference scheme with unconditional stability [26]. In this method the solution of equation (13) at the point $P_{j, k}$ (Fig. 3) was approximated with the use of 15 values in the rectangle with corners $P_{j-2, k-1}$, $P_{j+2, k-1}, P_{j-2, k+1}$, and $P_{j+2, k+1}$.

To approximate the spatial derivative the finite difference approximation calculated along the lines $k+1$ and $k-1$ with weight factor $\theta$ and the line $k$ with (1-20) (see Fig. 3) were averaged. For example $x^{\prime \prime \prime \prime}=\left(1+\theta \delta_{t}^{2}\right) \delta_{y}^{4} P_{j, k} / h^{4}$ at point $P_{j, k}$ where $\delta_{y}^{4}$ and $\delta_{t}^{2}$ are given by equation (47) was approximated. Similarly, for temporal derivative, the lines $j+1$ and $j-1$ were weighted with factor $\beta$ and the line $j$ with $(1-2 \beta)$ and then averaged. Values of $\theta=0.4$ and $\beta=1 / 6$ were used in the numerical scheme which converged [26] with an approximation of order $O\left(h^{4}\right)$. The numerical value for $h$ was taken as $0.05 \mathrm{~m}$ and that of $\Delta t$ was $0.001 \mathrm{~s}$, however, simulation plots are shown with $h=0.5 \mathrm{~m}$ and $\Delta t=0.02 \mathrm{~s}$ for clarity and better understanding.

$$
\begin{aligned}
\delta_{y}^{4} P_{j, k} & =P_{j-2, k}-4 P_{j-1, k}+6 P_{j, k}-4 P_{j+1, k}+P_{j+2, k} \\
\delta_{t}^{2} P_{j, k} & =P_{j-1, k}-2 P_{j, k}+P_{j+1, k}
\end{aligned}
$$

The beam problem was solved for different initial conditions. In the first case the initial condition of equation (48) was taken as a static displacement due to a uniformly distributed load of $p=1 \mathrm{~kg} / \mathrm{m}$. This resembles the first mode of vibration of the linear model of the beam. In other words, the dynamics of 


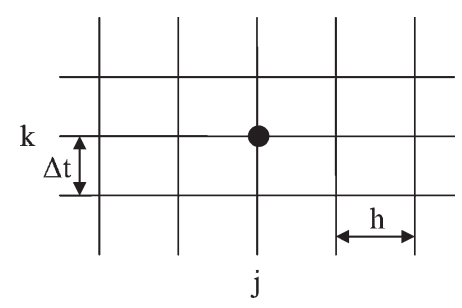

Fig. 3 Implicit finite difference mesh

Table 1 Parameter values

\begin{tabular}{lll}
\hline Parameter & Description & Value \\
\hline$E I$ & Rigidity of the beam & $291.6667 \mathrm{~N} \mathrm{~m}^{2}$ \\
$m$ & Mass per unit length & $1.3850 \mathrm{~kg} / \mathrm{m}$ \\
$L$ & Length of the beam & $5 \mathrm{~m}$ \\
$c$ & Damping coefficient & $1 \%$ of critical \\
$p$ & Initial load on the beam & $1 \mathrm{~kg} / \mathrm{m}$ \\
$x$ & Displacement of the beam & State variable \\
\hline
\end{tabular}

the beam execute the condition of a suddenly released load. Therefore, in this case the initial conditions are

$$
\left\{\begin{array}{l}
x(y, 0)=p y\left(y^{3}+L^{3}-2 L y^{2}\right) / 24 E I \\
\dot{x}(y, 0)=0
\end{array}\right.
$$

Figure 4, shows the uncontrolled displacement plot of the beam. The amplitude of vibration decreases with time due to a 1 per cent mass proportional damping. The displacement amplitude of the beam was observed to be $0.024 \mathrm{~m}$ after $10 \mathrm{~s}$ of vibration. The goal of this paper is to bring this beam vibration to zero.

A beam with controllers was simulated with a weighing matrix $\mathbf{Q}$ (see equation (16)) of

$$
\mathbf{Q}=\left[\begin{array}{cc}
100 & 36.8 \\
36.8 & 15
\end{array}\right]
$$

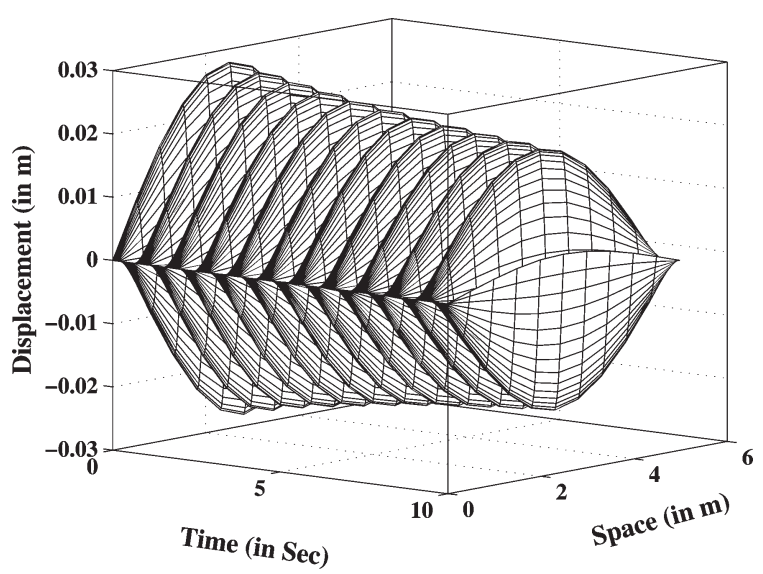

Fig. 4 Uncontrolled displacement time history and the control gain, $k$, was set to ten. Since, no relative importance to any point on spatial domain $y \in[0, L]$ was given, $r(y)$ was taken to be a constant. Therefore, the simplified expression for the controller shown in equation (28) could be applied.

The controlled displacement and time histories for the case of the continuous controller are shown in Fig. 5 and Fig. 6 respectively. From the controlled displacement (Fig. 5) plot it is evident that the vibration of the uncontrolled beam is bought to zero within $4 \mathrm{~s}$ with a little overshoot. This overshoot can be controlled by monitoring the $\mathbf{Q}$ matrix. The control force (Fig. 6) input is less than $0.5 \mathrm{~N}$. It should be noted that the control force flow is similar to the state flow of the system and dies down to zero as the states reach their goals.

For the simulations with the discrete controller, the same control gain $(k=10)$ and weighing matrix Q were taken. The tolerance value was set to tol $=5 \times 10^{-4}$. After switching the control gain, $\mathrm{K}=\operatorname{diag}\left(k_{1}, \ldots, k_{N}\right)$ was used, and values of

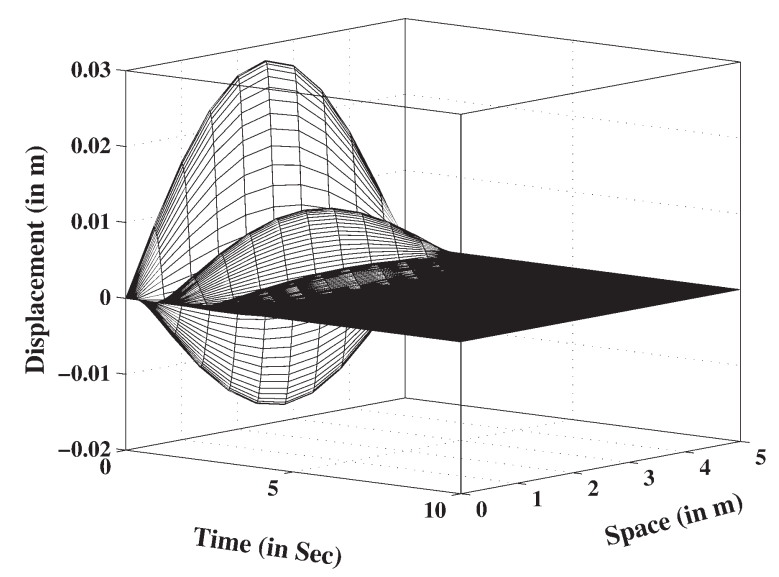

Fig. 5 Controlled displacement profile

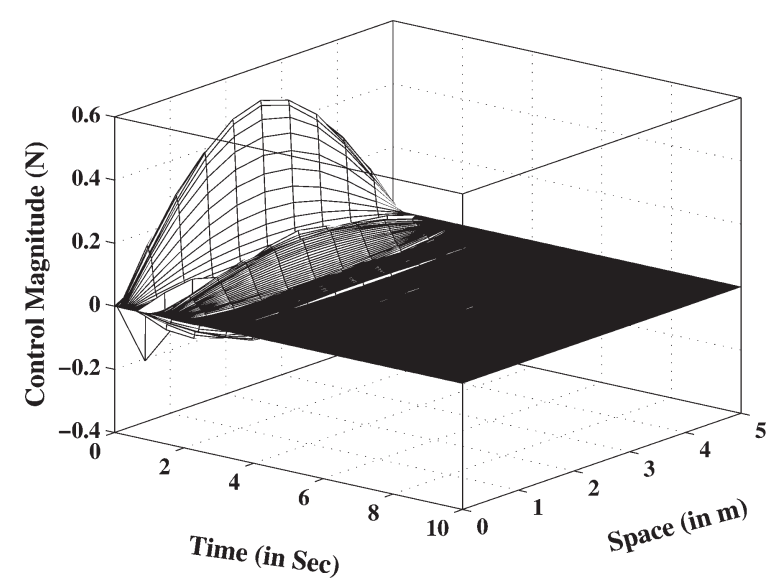

Fig. 6 Control time history 
$\omega_{n}=2.5, \zeta_{n}=0.5$ for $n=1, \ldots, N$ were selected. The parameters $w_{1}=\ldots=w_{N}=0.05 \mathrm{~m}$ and it was assumed that $r_{1}=\ldots=r_{N}$. With this assumption, the simplified expression for the controller (equation (42)) could be used when $\|I\|_{2}>$ tol. Because of this no numerical values for $r_{1}, \ldots, r_{N}$ were required.

Simulations were run for different numbers of control actuators (assumed to be equally spaced in the spatial domain) for $10 \mathrm{~s}$. It was observed that the beam vibrations significantly decrease within this time. Switching between the two control algorithms resulted in a sudden jump in the plots. To minimize these sudden jumps and to smooth the control force provided by the actuators two tolerance values to $_{1}$ and $t o l_{2}$ were set. The idea is, before reaching $t o l_{1}$ the original discrete controller is used and after $t o l_{2}$ the point controller takes over. Between tol $_{1}$ and $t_{0} l_{2}$ a convex combination (equation (49)) of the control algorithms based on $\|I\|_{2 \text { present }}$ (value of $\|I\|_{2}$ at the current position) value is used

$$
\bar{u}_{n}=(1-\alpha) \bar{u}_{n 1}+\alpha \bar{u}_{n 2}
$$

where $\alpha=\left(\right.$ tol $\left._{1}-\|I\|_{2 \text { present }}\right) /\left(\right.$ tol $_{1}-$ tol $\left._{2}\right)$ and $\bar{u}_{n 1}, \bar{u}_{n 2}$ are controllers based on the original and revised goals respectively. tol $_{2}$ was taken to be $1 \times 10^{-6}$.

Simulations were also run with a linear beam model and a linear controller, a non-linear beam with one actuator situated at the centre of the beam, and a non-linear beam with three equally spaced actuators. The controller for the linear model was designed using the techniques presented in section 3 . Figures 7 and 8 are comparisons of the displacement and velocity norms obtained in the simulation studies. It is observed that non-linear control with a single actuator situated at the centre of the beam can reduce the beam vibration but the performance is worse than the other studied approaches. This

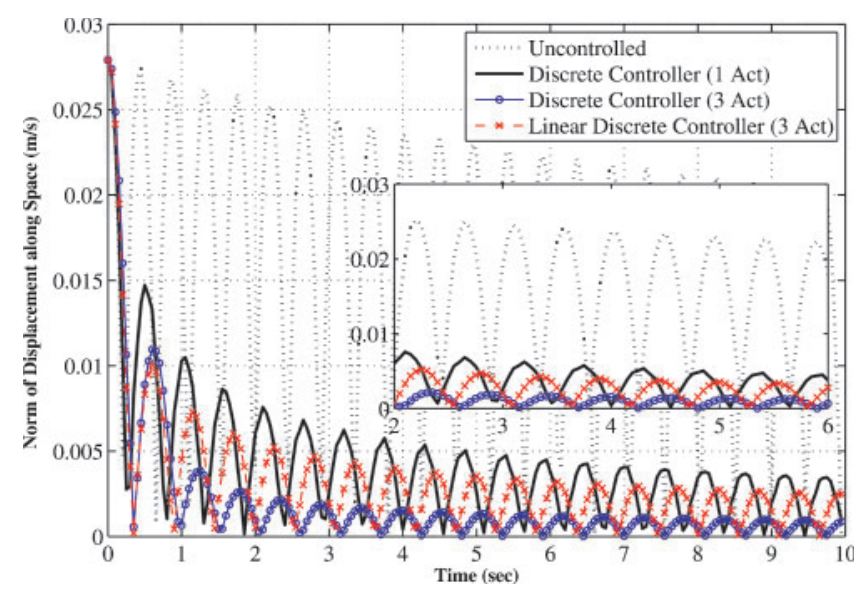

Fig. 7 Comparison of the displacement norm

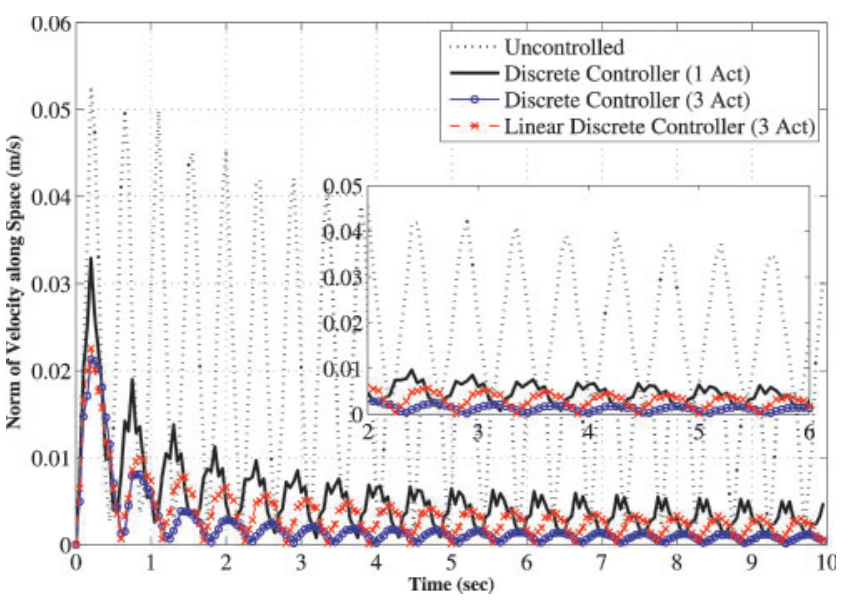

Fig. 8 Comparison of the velocity norm

highlights the point that multiple actuators are required to reduce the beam vibration. The performance of the linear controller on linear beam model with three actuators is not as good as the non-linear controller with three actuators. Therefore, the nonlinear controller is better for beams and the nonlinear beams should be controlled using non-linear control techniques. Henceforth, all the discussed simulations concern the use of a non-linear controller.

Figure 9 shows that the control goal (with three actuators) is achieved within $6 \mathrm{~s}$ of the initial disturbance with a little overshoot. It is evident from Fig. 10 that there is a smooth transition between the controllers. The switch between controllers occurs near every zero crossing of the displacement profile of the beam. Selecting a sufficiently low value for $t o l_{1}$ within achievable limits of the control magnitude, the jump can be minimized. Moreover, this behaviour can be further reduced by increasing the number of actuators and taking the actuator dy-

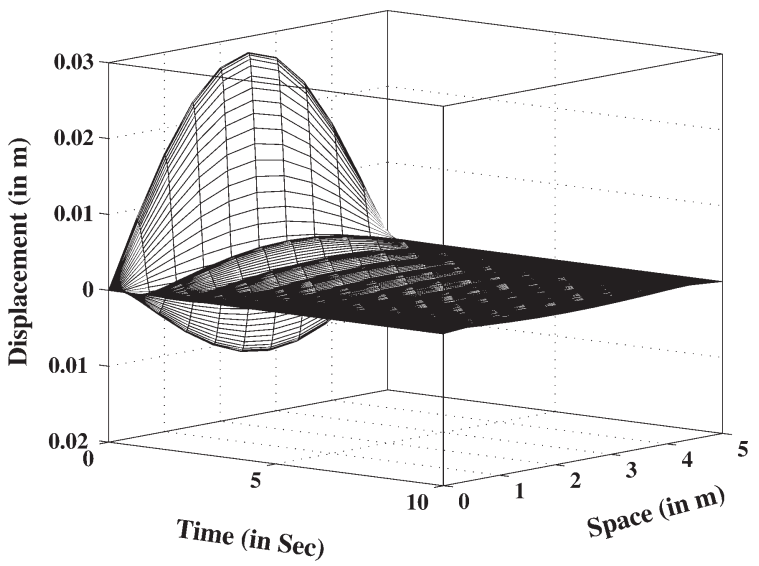

Fig. 9 Evolution of the displacement (state) profile using three actuators 


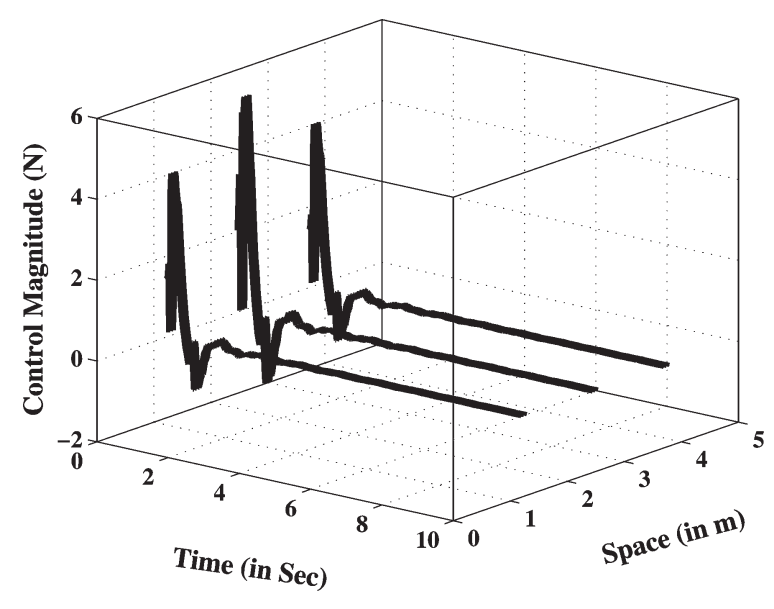

Fig. 10 Control time history when using three actuators

namics into consideration (actuator dynamics are not taken into consideration in this work). Again a small amount of overshoot in the state time histories can be observed.

Simulations were also carried out for the situation where more controllers are used. The figures for these simulations are not shown for brevity, only a comparison of the velocity norm (Fig. 11) and integral error function (Fig. 12) (given by equation (16)) are reported.

Figure 11 shows a comparison of the velocity profile of the beam for uncontrolled and controlled cases (and continuous controller, discrete controller with three actuators, and discrete controller with six actuators). In this figure the norm of the velocity is plotted with respect to the time $t$ which is a measure of the kinetic energy in the system. It can be seen that whereas the velocity state for the continuous controller reaches zero, it does not exactly go to zero for discontinuous controllers. This is because the final controller is a point controller and it is designed

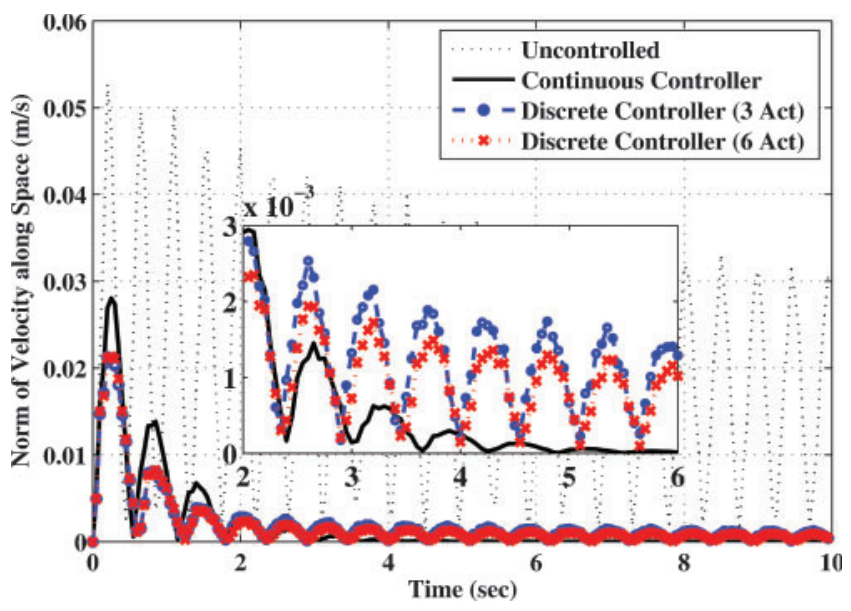

Fig. 11 Comparison of the velocity norm along space

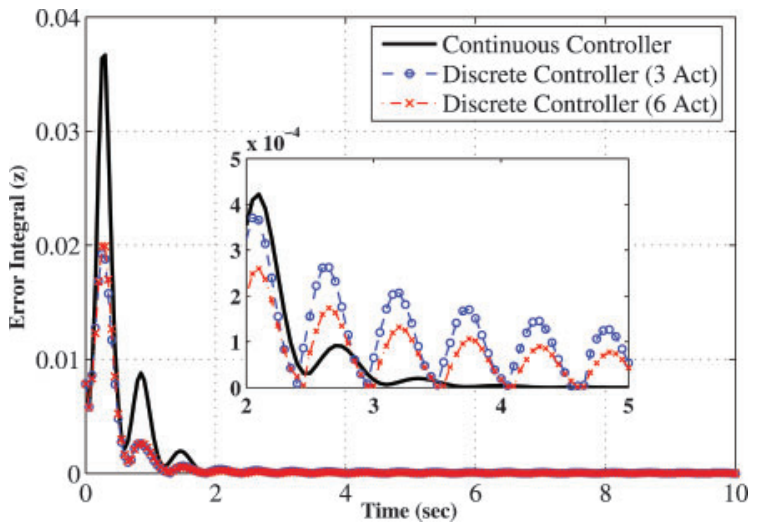

Fig. 12 Integral error time history

to act only on the region with which it is in contact, therefore the velocity of the beam does not reach zero everywhere in $y \in[0, L]$, but only in the range of the controller.

Figure 12 shows the dynamics of $Z(t)$ (see equation (16)) with time for a continuous controller, a discrete controller with three actuators, and a discrete controller with six actuators. Figure 12 clearly shows that the error function reaches zero within $4 \mathrm{~s}$ of the onset of vibration for the continuous controller whereas the discrete controllers are still suffering from vibration albeit at reduced values. With six actuators it can be seen that the wavy nature is substantially smaller and the goal of a zero state is met with a smaller overshoot compared to the discrete controller with three actuators case. The control effectiveness is observed to be higher and subsequently the magnitudes of the controllers are nearly half of that required for the three-actuator controller. Another important observation is that the curve for the discrete controller with six actuators is more close to that of the continuous case. This is because as the number of actuators increases the controller increasingly approaches being a continuous controller. Since $Z(t)$ is a function of both states, the exponential decay of the peaks shows that both states have reached their goals.

In the next sequence of experiments random initial profiles for the beam were generated and the same controller with same parameters were used to minimize beam non-linear vibration. Due to space limitations only plots for the states and the controller force are presented.

\subsection{Random initial profile generation}

Random initial profiles were generated using a Fourier series with a $L_{2}$-norm bound. The funda- 
mental idea in this type of state profile generation is first to define the domain of interest based on $L_{2}$ norm bounds for the state and one or more of its spatial derivatives. Then, these profiles are approximated by an expansion of a series of sine functions with a finitely large number of terms such that it satisfies $\langle x(y, 0), x(y, 0)\rangle \leqslant\left\langle x_{m}, x_{m}\right\rangle$ and $\left\langle x^{\prime \prime}(y, 0)\right.$, $\left.x^{\prime \prime}(y, 0)\right\rangle \leqslant\left\langle x_{m}^{\prime \prime}, x_{m}^{\prime \prime}\right\rangle$, where $\langle x, x\rangle \triangleq \int_{0}^{L} x^{2}(y) \mathrm{d} y$. In the random profile generation $\left\langle x_{m}, x_{m}\right\rangle=1.25$ and $\left\langle x_{m}, x_{m}\right\rangle=0.1$ and 50 terms were taken in the Fourier expansion. The $L_{2}$-norm was taken to keep the initial profile of the beam within certain energy bounds as well as to avoid too much (unrealistic) waviness in the profiles. Details of random profile generation can be found in [22].

The simulations with random initial profiles were also run for $10 \mathrm{~s}$. It was observed that the controlled beam vibration reduces within this time. Figure 13 shows the uncontrolled displacement time history of the beam with a random initial profile. Whereas the uncontrolled beam displacement keeps on vibrating even after $10 \mathrm{~s}$ of motion with an amplitude of $0.015 \mathrm{~m}$, the controlled vibration (Fig. 14) reduces within $3 \mathrm{~s}$ of the onset of the vibration. The maximum control force was less than $8 \mathrm{~N}$. Moreover, the control force converges to its final zero value everywhere in $y \in[0, L]$ as the beam stops vibrating (see Fig. 15).

The random initial displacement profile resembles the second mode of vibration of the beam (linearized dynamics). The wavy nature of the uncontrolled displacement (Fig. 13) is greater and therefore an ATD approach is hard to implement (in this approach high-frequency dynamics are neglected). However, the controlled displacement plot (Fig. 14) shows the efficiency of the optimal dynamic inversion approach. Four actuators were taken to simu-

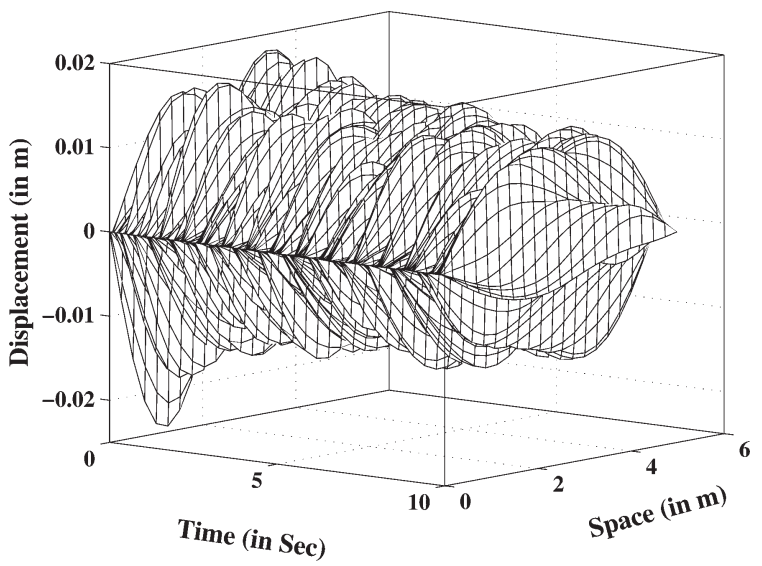

Fig. 13 Uncontrolled displacement time history (random profile)

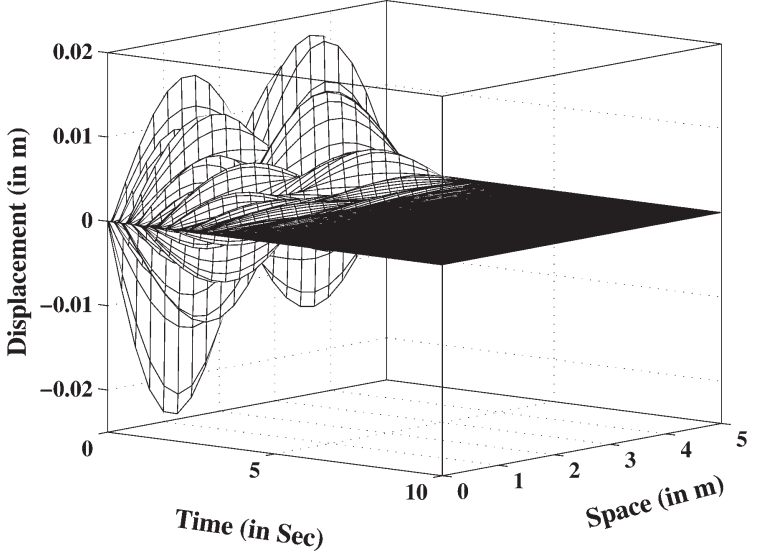

Fig. 14 Controlled displacement profile (random profile)

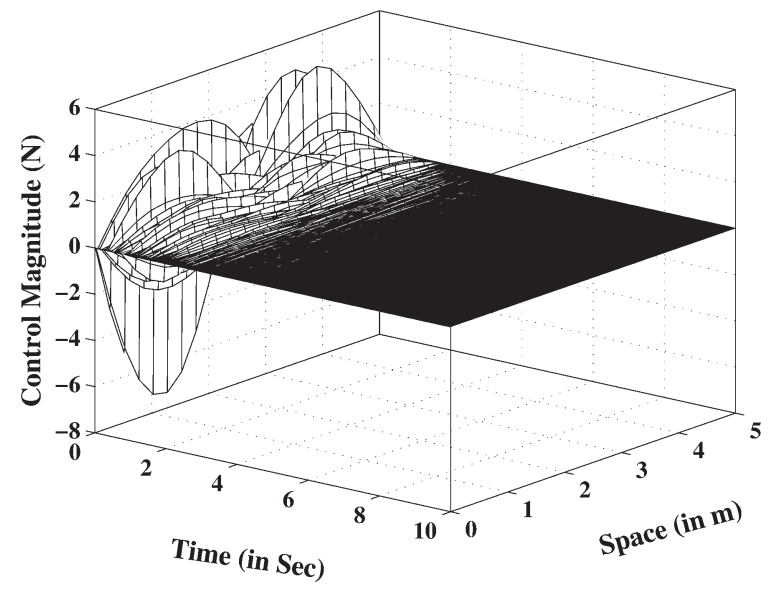

Fig. 15 Control time history (random profile)

late with the discrete actuators. It is evident from Fig. 16 that the displacement state has reached its goal. Figure 17 shows the control force required to minimize the beam vibration. Simulations were also run for the three-actuators case and similar conclu-

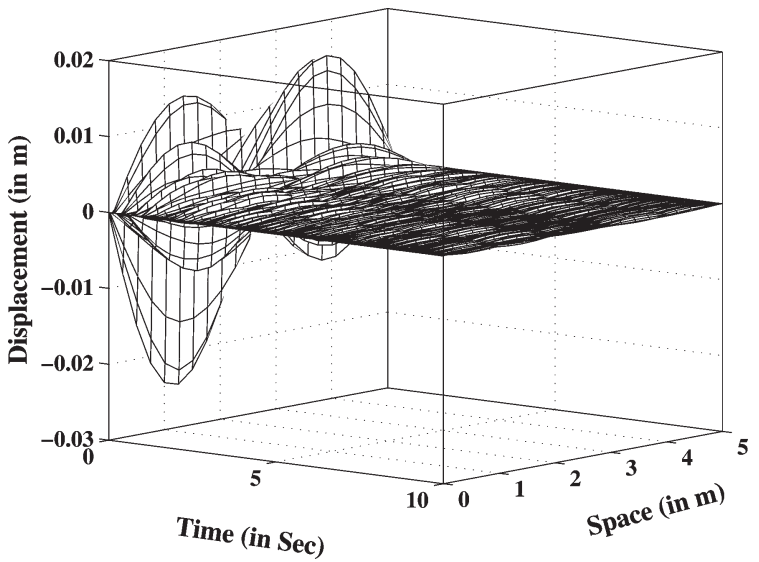

Fig. 16 Evolution of the displacement (state) profile with four actuators (random profile) 


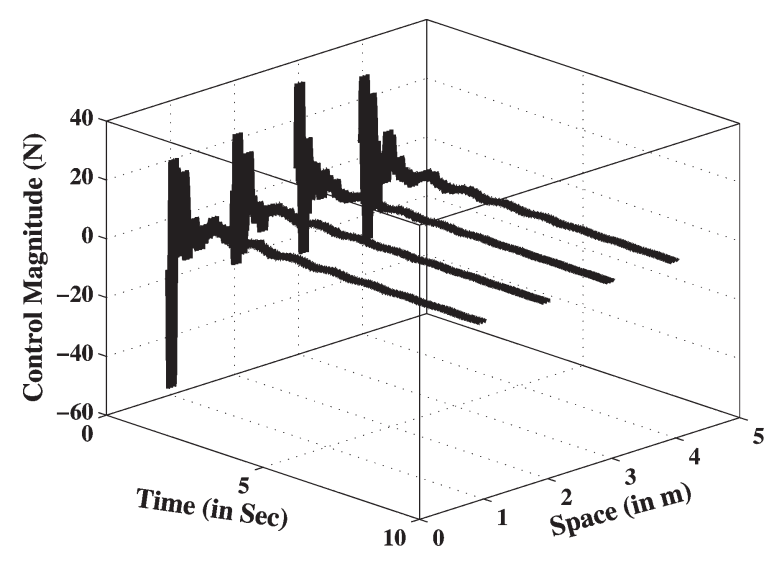

Fig. 17 Control time history for four actuators (random profile)

sions were drawn as those discussed in the first case. Figure 18 shows a comparison of the velocity profile of the beam for the uncontrolled and controlled cases (continuous controller, discrete controller with three actuators, and discrete controller with four actuators). The same velocity norm as the one discussed in the first case was taken i.e. the norm of the velocity is plotted with respect to the time $t$. It is evident from the figure that for continuous control the velocity reaches zero before $4 \mathrm{~s}$ but for discrete cases it vibrates with reduced magnitude.

The first initial condition corresponds to the first mode of vibration of the beam and the second random initial condition represents the second mode of vibration of the beam. Both of these modes are effectively suppressed and reduced to a zero value within a short time interval (without demanding an infeasible amount of control energy). Therefore, it can be concluded that the used technique (optimal dynamic inversion) can effectively be used in civil engineering problems to suppress structural vibrations.

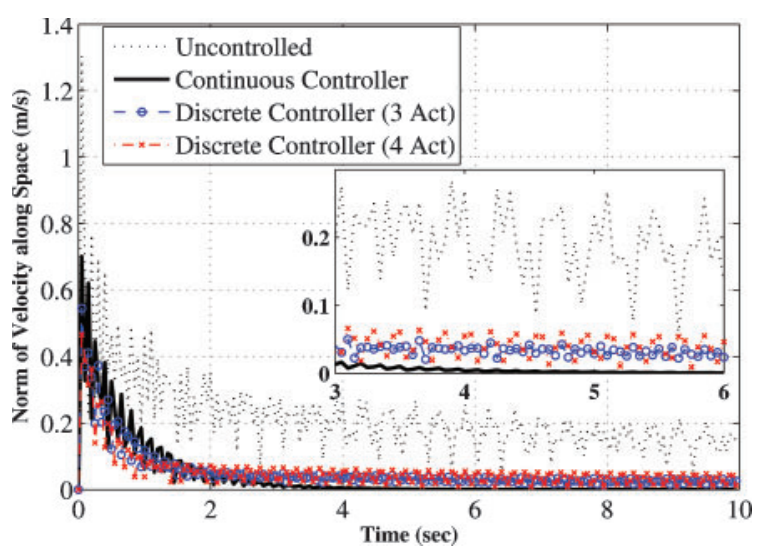

Fig. 18 Comparison of the norm of the velocity along space (random profile)

\section{CONCLUSIONS}

Simply supported non-linear Euler-Bernoulli beam vibration under different initial conditions has been shown to be minimized using newly developed optimal dynamic inversion approach. Both continuous and discrete actuators have been considered. The method directly uses the system non-linear PDE to develop the controller force based on the error between actual and desired state profiles (in the $L_{2}$ norm sense). Here the error caused in modal truncation while obtaining the reduced-order system does not arise. Therefore, errors due to controller and observer spillovers can be avoided. Two representative different initial conditions are taken to show the effectiveness of the present approach (similar results have been obtained in the numerous simulation studies using various initial conditions). The PDEs have been solved using an unconditionally stable implicit finite difference scheme. Furthermore, the formulation has good practical significance since it can be used to implement a set of discrete controllers with relative ease. It can also be implemented online for real-time implementation since a closed-form solution is obtained for the control variable.

\section{ACKNOWLEDGEMENT}

The authors thank Professor Ananth Ramaswamy, Department of Civil Engineering, Indian Institute of Science, Bangalore, India for discussions on the physics of the studied problem.

\section{REFERENCES}

1 Nayfeh, A. H. and Pai, P. F. Linear and nonlinear structural mechanics, 2004 (Wiley, Berlin).

2 Kar, I. N., Seto, K., and Doi, F. Multimode vibration control of a flexible structure using $H_{\infty}$ based robust control. IEEE/ASME Trans. Mechatron., 2000, 5(1), 23-31.

3 Yang, J. B., Jiang, L. J., and Chen, D. C. H. Dynamic modeling and control of a rotating EulerBernoulli beam. J. Sound Vib., 2004, 274(3-5), 863-875.

4 Jalili, N. An infinite dimensional distributed base controller for regulation of flexible robot arms. Trans. ASME, J. Dyn. Syst., Meas. Control, 2001, 123, 712-718.

5 Lasiecka, I. Control of systems governed by partial differential equations: a historical perspective. In Proceedings of the 34th IEEE Conference on Decision and control, New Orleans, LA, 1995, pp. 2792-2796 (IEEE, Piscataway, NJ). 
6 Padhi, R. and Faruque, Ali Sk. An account of chronological developments in control of distributed parameter systems. Ann. Rev. Control (in press). DOI: 10.1016/j.arcontrol.2009.01.003.

7 Burns, J. and King, B. B. Optimal sensor location for robust control of distributed parameter systems. In Proceedings of the 33rd IEEE Conference on Decision and control, 1994, pp. 3967-3972 (IEEE, Florida, USA).

8 Carlos Mazzilli, E. N., Soares, M. E. S., and Baracho Neto, O. G. P. Non-linear normal modes of a simply supported beam: continuous system and finite-element models. Comput. Struct, 2004, 82, 2683-2691.

9 Fung, R. F., Wu, J. W., and Lu, P. Y. Adaptive boundary control of an axially moving string system. Trans. ASME, J. Vib. Acoust., 2002, 124, 435-440.

10 Wu, Z. and Yildizoglu, S. E. Distributed parameterdependent modeling and control of flexible structures. Trans. ASME, J. Dyn. Syst. Meas. Control, 2005, 127, 230-239.

11 Gunzburger, M. D., Peterson, J. S., and Shadid, J. N. Reduced order modeling of time dependent PDEs with multiple parameters in the boundary data. Comput. Methods Appl. Mech. Engng, 2007, 196(4-6), 1030-1047.

12 Holmes, P., Lumley, J. L., and Berkooz, G. Turbulence, coherent structures, dynamical systems and symmetry, 1996 (Cambridge University Press, Cambridge, UK).

13 Yang, B. Transfer function of constrained/combined one dimensional continuous dynamic systems. J. Sound Vib., 1992, 156(3), 425-443.

14 Yang, B. and Mote, C. D. Active vibration control of axially moving string in S domain. J. Appl. Mech., 1991, 58(1), 189-196.

15 Tadi, M. Nonlinear feedback control of slewing beams. Comput. Methods Appl. Mech. Engng, 2006, 195(1-3), 133-147.

16 Canbolat, H., Dawson, D., Rahn, C., and Vedagarbha, P. Boundary control of a cantilevered flexible beam with point-mass dynamics at the free end. Mechatronics, 1998, 8, 163-186.

17 Pang, S. T., Sao, T. C., and Bergman, L. A. Active and passive damping of Euler-Bernoulli beam and their interaction. Trans. ASME, J. Dyn. Syst., Meas. Control, 1993, 115, 379-384.

18 Curtain, R. F. and Zwart, H. J. An introduction to infinite dimensional linear systems theory, 1995 (Springer-Verlag, New York, NY).

19 Bameih, B. The structure of optimal controllers of spatially-invariant distributed parameter systems. In Proceedings of the IEEE Conference on Decision and control, 1997, pp. 1056-1061 (IEEE, San Diego, California, USA).

20 Sun, D. and Mills, J. K. Control of a rotating cantilever beam using a torque actuator and a distributed piezoelectric polymer actuator. Appl. Acoustics, 2002, 63, 885-899.
21 Bourquin, F., Branchet, B., and Collet, M. Computational methods for the fast boundary stabilization of flexible structures. Part 1: the case of beams. Comput. Methods Appl. Mech. Engng, 2007, 196(4-6), 988-1005.

22 Padhi, R. and Balakrishnan, S. N. An optimal dynamic inversion approach for controlling a class of one-dimensional nonlinear distributed parameter systems. In Proceedings of the American Control Conference, 2006, pp. 57-62 (IEE, Minnesota, USA).

23 Slotine, J. E. and Li, W. Applied nonlinear control, 1991 (Prentice Hall, New Jersey).

24 Khalil, H. K. Nonlinear systems, 1996 (Prentice Hall, New Jersey).

25 Bryson, A. E. and Ho, Y. C. Applied optimal control, 1975 (Taylor and Francis, London).

26 Crandall, S. H. Optimum recurrence formulas for a fourth order parabolic partial differential equation. J. Assoc. Comput. Mach., 1957, 4(4), 467-471.

\section{APPENDIX}

\section{Proof of convergence of continuous controller}

Before it is possible to prove the convergence of control solution, the system dynamics when the states reach their goals need to be defined. When $x \rightarrow 0$ and $\dot{x} \rightarrow 0$, the system dynamics become

$$
\ddot{x}^{*}+\frac{c}{m} \dot{x}^{*}+f\left(x^{*}, x^{* \prime}, x^{* \prime \prime}, \ldots\right)=\frac{1}{m} u^{*}(y, t)
$$

where $\ddot{x}^{*}=0$ and $\dot{x}^{*}=0$ since the goals of states is to reach zero, $f\left(x, x^{\prime}, x^{\prime \prime}, \ldots\right)$ denotes the non-linear stiffness part of the system dynamics in equation (13). Therefore, $f\left(x, x^{\prime}, x^{\prime \prime}, \ldots\right)$ is continuous and $f(x$, $\left.x^{\prime}, x^{\prime \prime}, \ldots\right) \rightarrow f\left(x^{*}, x^{* \prime}, x^{* \prime \prime}, \ldots\right)=f^{*}$ as $x \rightarrow 0$ and $\dot{x} \rightarrow 0$. The final condition of $f\left(x, x^{\prime}, x^{\prime \prime}, \ldots\right)$ depends on $\left(x^{*}\right.$, $\left.x^{* \prime}, x^{* \prime \prime}, \ldots\right)$. As $x \rightarrow 0$ and $\dot{x} \rightarrow 0$, then it is possible to make by choice $\left(x^{*}, x^{* \prime}, x^{* \prime \prime}, \ldots\right) \rightarrow 0$, which makes $f^{*} \rightarrow 0$.

\section{Theorem 1.}

$u(t, y)$ in equation (28) converges to zero when $x(y, t) \rightarrow 0$ and $\dot{x}(y, t) \rightarrow 0, \forall y_{0} \in[0, L]$.

Proof.

Notice that at any point $y_{0} \in[0, L]$, the control solution in equation (28) can be written as

$$
u\left(y_{0}, t\right)=\frac{m[\gamma]_{y_{0}}\left(q_{12} x\left(y_{0}\right)+q_{22} \dot{x}\left(y_{0}\right)\right)}{\left[\int_{0}^{L}\left(q_{12} x+q_{22} \dot{x}\right)^{2} \mathrm{~d} y\right]_{y_{0}}}
$$


where $[\cdot]_{y_{0}}$ denotes the expression as evaluated at $y_{0}$. It is necessary to analyse this solution for the case when $x(y, t)=0$ and $\dot{x}(y, t)=0$ for all $y \in[0, L]$. Without loss of generality, the case can be analysed in the limit when $x(y, t) \rightarrow 0$ and $\dot{x}(y, t) \rightarrow 0$, for $y \in\left[y_{0}-\varepsilon / 2, y_{0}+\varepsilon / 2\right] \subset[0, L], \varepsilon \rightarrow 0$, and $x(y, t)=0$ and $\dot{x}(y, t)=0$, everywhere else. In such a limiting case, let the control value be denoted by $\bar{u}\left(y_{0}, t\right)$, which is given by zero everywhere except at the interval $y \in\left[y_{0}-\varepsilon / 2\right.$, $\left.y_{0}+\varepsilon / 2\right] \subset[0, L], \varepsilon \rightarrow 0$, where the states are sufficiently small. Because of this, the system can be assumed to behave linearly at this interval. This assumption leads to a linear relationship between $x\left(y_{0}\right)$ and $\dot{x}\left(y_{0}\right)$, i.e. $\dot{x}\left(y_{0}\right)=a x\left(y_{0}\right)$, where $a$ is a constant.

Substituting $\dot{x}\left(y_{0}\right)=a x\left(y_{0}\right)$ in the limit (equation (52)), it is possible to obtain

$$
\begin{aligned}
& \bar{u}\left(y_{0}, t\right)=\frac{-m}{\left[\int_{y_{0}-\varepsilon / 2}^{y_{0}+\varepsilon / 2}(g)^{2} \mathrm{~d} y\right]} \\
& {\left[\int_{y_{0}-\varepsilon / 2}^{y_{0}+\varepsilon / 2}\left\{\left(q_{11} x \dot{x}+q_{12} \dot{x}^{2}\right)-\left(q_{21} x+q_{22} \dot{x}\right)\left(\frac{c}{m} \dot{x}+f(t, x(y), \ldots)\right)+\frac{k}{2}\left([x \dot{x}] \mathbf{Q}[x \dot{x}]^{\mathrm{T}}\right)\right\}\left\{q_{12} x\left(y_{0}\right)+q_{22} \dot{x}\left(y_{0}\right)\right\}\right]}
\end{aligned}
$$

where $g$ represents $\left[\left(q_{12} x(y)+q_{22} \dot{x}(y)\right)\right]$. Therefore

$$
\begin{aligned}
& \bar{u}\left(y_{0}, t\right)=\frac{-m \times \varepsilon}{\left[g\left(y_{0}\right)\right]^{2} \times \varepsilon} \\
& \quad\left[\left\{\left(q_{11} x\left(y_{0}\right) \dot{x}\left(y_{0}\right)+q_{12} \dot{x}\left(y_{0}\right)^{2}\right)-\left(q_{21} x\left(y_{0}\right)+q_{22} \dot{x}\left(y_{0}\right)\right)\left(\frac{c}{m} \dot{x}\left(y_{0}\right)+f\left(t, x\left(y_{0}\right), \ldots\right)\right)+\frac{k}{2}\left(\left[x\left(y_{0}\right) \dot{x}\left(y_{0}\right)\right] \mathbf{Q}\left[x\left(y_{0}\right) \dot{x}\left(y_{0}\right)\right]^{\mathrm{T}}\right)\right\}\right. \\
& \left.\quad \times\left\{q_{12} x\left(y_{0}\right)+q_{22} \dot{x}\left(y_{0}\right)\right\}\right]
\end{aligned}
$$

Expanding the terms and cancelling the common terms in the numerator and the denominator

$$
\begin{aligned}
\bar{u}\left(y_{0}, t\right)=m[ & \frac{q_{11} x\left(y_{0}\right) \dot{x}\left(y_{0}\right)+q_{12} \dot{x}^{2}\left(y_{0}\right)}{q_{12} x\left(y_{0}\right)+q_{22} \dot{x}\left(y_{0}\right)}+\frac{k}{2} \frac{q_{11} x\left(y_{0}\right)^{2}+2 q_{12} x\left(y_{0}\right) \dot{x}\left(y_{0}\right)+q_{22} \dot{x}^{2}\left(y_{0}\right)}{q_{12} x\left(y_{0}\right)+q_{22} \dot{x}\left(y_{0}\right)} \\
& \left.-\left(\frac{c}{m} \dot{x}\left(y_{0}\right)+f\left(t, x\left(y_{0}\right), \ldots\right)\right)\right]
\end{aligned}
$$

The third term $(c / m) x\left(y_{0}\right)+f\left(t, x\left(y_{0}\right), \ldots\right) \rightarrow f^{*} \rightarrow 0$ as $x\left(y_{0}\right) \rightarrow 0$ and $\dot{x}\left(y_{0}\right) \rightarrow 0$. Therefore, in the limiting sense equation (51) simplifies to

$$
\begin{aligned}
\bar{u}\left(y_{0}, t\right)= & m \frac{q_{11} x\left(y_{0}\right) \dot{x}\left(y_{0}\right)+q_{12} \dot{x}^{2}\left(y_{0}\right)}{q_{12} x\left(y_{0}\right)+q_{22} \dot{x}\left(y_{0}\right)} \\
& +\frac{m k}{2} \frac{q_{11} x\left(y_{0}\right)^{2}+2 q_{12} x\left(y_{0}\right) \dot{x}\left(y_{0}\right)+q_{22} \dot{x}^{2}\left(y_{0}\right)}{q_{12} x\left(y_{0}\right)+q_{22} \dot{x}\left(y_{0}\right)}
\end{aligned}
$$

$$
\begin{aligned}
\bar{u}\left(y_{0}, t\right)= & \lim _{x\left(y_{0}\right) \rightarrow 0} m \frac{\left(q_{11} a+q_{12} a^{2}\right) x^{2}\left(y_{0}\right)}{\left(q_{12}+q_{22} a\right) x\left(y_{0}\right)} \\
& +\frac{m k}{2} \frac{\left(q_{11}+2 q_{12} a+q_{22} a^{2}\right) x^{2}\left(y_{0}\right)}{\left(q_{12}+q_{22} a\right) x\left(y_{0}\right)} \\
= & 0
\end{aligned}
$$

Equation (52) is limit in two variables $x\left(y_{0}\right) \rightarrow 0$ and $\dot{x}\left(y_{0}\right) \rightarrow 0$. It is assumed that the system states are

Moreover, the above discussion is true $\forall y_{0} \in[0, L]$. Hence, $u(y, t) \rightarrow 0$ as $x(y, t) \rightarrow 0$ and $\dot{x}(y, t) \rightarrow 0$, $\forall y_{0} \in[0, L]$. This shows the convergence of the 
proposed controller as $x(y, t) \rightarrow 0$ and $\dot{x}(y, t) \rightarrow 0$, $\forall y_{0} \in[0, L]$.

Therefore, combining both the results, the control action can be summarized as follows $u(y, t)$

$$
=\left\{\begin{array}{cc}
0 & \text { if } x=0, \dot{x}=0 \\
m \gamma\left(q_{12} x+q_{22} \dot{x}\right) / \int_{0}^{L}\left(q_{12} x+q_{22} \dot{x}\right)^{2} \mathrm{~d} y & \text { otherwise }
\end{array}\right.
$$

